\title{
Anisotropy of Stable Single Domain Ferrimagnetic Particles in a Rock Sample from Gyroremanent Magnetization and Comparison with Other Anisotropy Methods
}

\author{
Liam L. Belisleㄹ, David K. Potter ${ }^{1 *}$, Brendan C. Snow ${ }^{1,2}$, Allyson L. Shewchuk ${ }^{1}$ \\ ${ }^{1}$ Department of Physics, University of Alberta, Edmonton, Canada \\ ${ }^{2}$ CNOOC Petroleum North America ULC, Calgary, Canada \\ Email: *dkpotter@ualberta.ca
}

How to cite this paper: Belisle, L.L., Potter, D.K., Snow, B.C. and Shewchuk, A.L. (2019) Anisotropy of Stable Single Domain Ferrimagnetic Particles in a Rock Sample from Gyroremanent Magnetization and Comparison with Other Anisotropy Methods. Journal of Modern Physics, 10, 673-686. https://doi.org/10.4236/jmp.2019.106048

Received: April 28, 2019

Accepted: May 26, 2019

Published: May 29, 2019

Copyright $\odot 2019$ by author(s) and Scientific Research Publishing Inc. This work is licensed under the Creative Commons Attribution International License (CC BY 4.0).

http://creativecommons.org/licenses/by/4.0/

cC) (7) Open Access

\begin{abstract}
The orientation of stable single domain (SSD) ferrimagnetic particles in an igneous rock sample was determined by a sensitive technique utilizing gyroremanent magnetization (GRM). Components of GRM were measured in the sample upon exposure to an alternating field (AF) at various orientations in 3 orthogonal planes. The major components of GRM exhibited a $\sin (2 \theta)$ dependence on AF orientation in the respective perpendicular planes. This was in accordance with theory [1] and contrary to some previously reported experimental results on magnetic recording tape, which produced a distorted $\sin (2 \theta)$ dependence of the GRM [1]. The explanation is likely due to the SSD ferrimagnetic particles in the rock sample being more dispersed (less interacting) compared to the highly interacting SSD particles in the magnetic tape sample of the previous study. The GRM results were consistent with another remanence anisotropy method, anisotropy of isothermal remanent magnetization (AIRM). This method again measures the anisotropy of the remanence carrying ferrimagnetic particles, but the IRM is also acquired by larger multidomain (MD) particles as well as by the SSD particles. The results were also consistent with the visible rock anisotropy (petrofabric), the anisotropy of magnetic susceptibility (AMS), and the shear wave velocity anisotropy. A comparison of all the methods demonstrated that the fine SSD particles, which make up only a small proportion of the rock, were aligned in quite a similar orientation to that of the main rock forming minerals that constituted the bulk of the sample.
\end{abstract}

\section{Keywords}

Anisotropy, Gyroremanence, Isothermal Remanence, Magnetic Susceptibility, 


\section{Introduction}

Gyroremanent magnetization (GRM) is produced by exposing a sample containing an anisotropic distribution of fine ferro- or ferrimagnetic particles to a single alternating field (AF) application [1], or by exposing a sample containing an isotropic distribution of such particles to two successive applications of an AF [2]. GRM is theoretically only produced by stable single domain (SSD) remanence carrying particles [1]. These are the very particles that are most useful for both man-made recording processes, and for palaeomagnetic purposes for recording the magnitude and direction of the ancient geomagnetic field in rocks over geological time periods. By measuring the components of GRM produced when an AF is applied at various orientations in 3 orthogonal planes of a sample, it is possible to infer the orientation of the SSD particles in the sample. The method is the most sensitive remanence anisotropy method, and allows one to exclusively determine the anisotropic distribution of the SSD particles [3]. This means that GRM measurements represent a unique magnetic means of isolating the anisotropic distribution of SSD particles. This is important since an anisotropic distribution of these particles can deflect the natural remanent magnetization (NRM) in a rock sample away from the ancient field direction. This has potentially serious implications if the sample is used for palaeomagnetic purposes, since it could lead to errors in determining palaeomagnetic reconstructions and the behaviour (i.e., palaeointensity and direction) of the Earth's ancient magnetic field. In contrast to GRM anisotropy, other anisotropy of magnetic remanence (AMR) methods may include remanence components from any multidomain (MD) ferro- or ferrimagnetic particles present in the sample [3] in addition to the components from the SSD particles. The MD components are generally less stable over geological timescales.

A key aim of the present paper is to show that GRM measurements can readily determine the anisotropy of SSD particles in a rock sample that was previously considered "quasi-isotropic" from acoustic anisotropy methods [4]. Previous GRM anisotropy studies have investigated magnetic recording tapes [1], dispersed particles from magnetic recording tapes [5], a weakly anisotropic rock sample with no visible anisotropy (petrofabric) [6], or a more strongly anisotropic rock sample, again with no visible petrofabric [3]. In the present paper the rock sample exhibited a clearly visible petrofabric arising from the alignment of the larger mineral grains (plagioclase and quartz) that constituted the bulk of the sample. This allowed us to determine whether the anisotropy of the SSD particles (which constitute a small fraction of the rock sample) from our GRM measurements was related to the overall observed petrofabric of the main rock forming minerals.

We also undertook 3 other quantitative anisotropy methods in order to help 
verify the GRM anisotropy results of the SSD particles, and also to compare the SSD particle anisotropy with the overall anisotropy of the larger minerals comprising the bulk of the rock sample. These 3 methods were as follows:

- Anisotropy of isothermal remanent magnetization (AIRM), which determines the anisotropy of all the remanence carrying particles. This not only includes the SSD particles but also the larger MD particles.

- Anisotropy of magnetic susceptibility (AMS), which measures the combined AMS of all the minerals in the rock sample, including the main rock forming diamagnetic minerals (plagioclase and quartz), any paramagnetic minerals, as well as the remanence carrying ferrimagnetic minerals. In the present sample the AMS is dominated by the small fraction of ferrimagnetic particles.

- Anisotropy of shear-wave velocity. This mainly determines the anisotropy of the bulk matrix minerals (in this case mainly plagioclase and quartz).

The magnetic anisotropy methods (GRM, AIRM and AMS) allow a full 3 dimensional (3D) anisotropy to be measured on a single rock core plug sample. Most other anisotropy techniques require several samples oriented in different directions in order to generate 3D anisotropic information, since certain measurements can only be made in 1 dimension (1D) on a core plug (e.g., fluid permeability measurements), or in 2 dimensions (2D). The experimental set up for the shear wave velocity measurements only allows $2 \mathrm{D}$ anisotropy to be determined in one particular plane, as detailed in the Methods section below.

\section{Sample}

The rock sample, VOLC-B-Y, was taken from the Flin Flon Belt of the Trans-Hudson Orogeny in eastern Saskatchewan, west of the town of Flin Flon, Manitoba, and is from the Palaeoproterozoic Era (1.6 - 2.5 billion years ago). This area has been studied for some time due to the economic potential of the mineral deposits found nearby [4]. The cylindrical rock sample was $2.5 \mathrm{~cm}$ in diameter and $2 \mathrm{~cm}$ in length and is shown in Figure 1. The figure shows the sample both with and without the arbitrary sample axes $(X, Y, Z)$ superimposed. The $+X$ direction has a declination of $0^{\circ}$ (or $360^{\circ}$ ) and an inclination of $0^{\circ}$, the $+Y$ direction has a declination of $90^{\circ}$ and an inclination of $0^{\circ}$, and the $+Z$ direction has a declination of $0^{\circ}$ and an inclination of $90^{\circ}$.

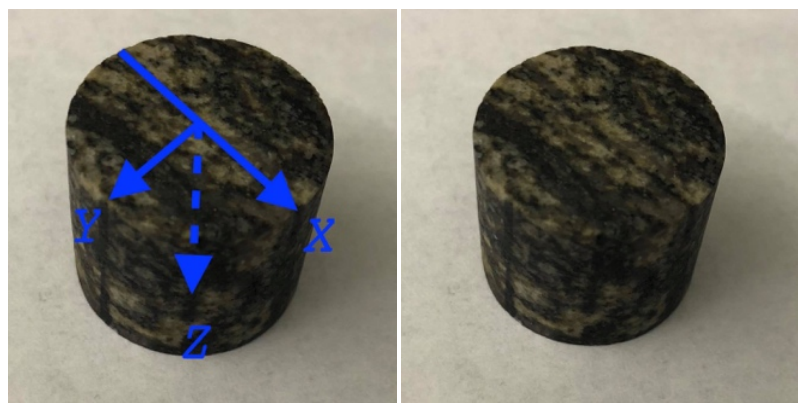

Figure 1. Sample VOLC-B-Y with (left) and without (right) the sample axes labeled. The directions of the arrows in the left hand figure indicate the $+X,+Y$ and $+Z$ directions. 
The sample had a mass of $28.33 \mathrm{~g}$ and the following mineralogical composition: $61.2 \%$ plagioclase, $22.5 \%$ quartz, $8.3 \%$ biotite, $1.9 \%$ opaque minerals [4]. The ferrimagnetic particles, representing a very small fraction of the rock sample, are likely to be magnetite or titanomagnetite based on some initial tests, including remanence acquisition curves. We did not determine the Curie temperature of the ferrimagnetic particles for this sample as we did not want to heat the sample in case this induced chemical changes. The previous study by Cholach et al. [4] described this rock sample as being "quasi-isotropic" from their acoustic anisotropy studies. However, all our experimental results will demonstrate that the sample is significantly anisotropic.

\section{Methods}

\subsection{Gyroremanent Magnetization (GRM) Anisotropy}

This method applied an AF to the static sample at various orientations in 3 orthogonal planes [3]. This was achieved using an AF demagnetizer coil housed within a mumetal shield. The latter shielded the sample from the Earth's field. The sample was subjected to an AF of $60 \mathrm{mT}$ at increments of $15^{\circ}$ within a $180^{\circ}$ range (from $\theta=-90^{\circ}$ to $90^{\circ}$ ) in a particular plane (Figure 2). After the AF application, the sample was removed from the AF coil and the GRM components $(x, y$ and $z$ ) were measured in a spinner magnetometer. Prior to each AF application the sample was tumble AF demagnetized in $80 \mathrm{mT}$ and the remaining remanence measured, and subtracted from the subsequent GRM measurement. The demagnetizing field was higher than the AF used to impart the GRM in order to remove as much remanence as possible prior to each GRM production step. 13 pairs of magnetized and demagnetized values were obtained in the 3 orthogonal planes $(X Y, Y Z$, and $Z X)$ giving a total of 39 measurements of GRM. For each measured plane the first listed axis was oriented at $\theta=0^{\circ}$ and the second axis oriented at $\theta=-90^{\circ}$ or $90^{\circ}$. The GRM method is extremely sensitive,

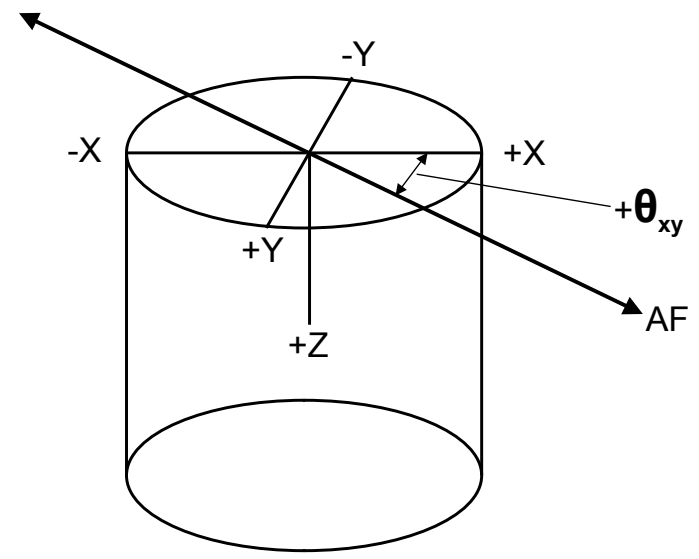

Figure 2. Example orientation of the AF axis with respect to the sample axes. The example shows the AF axis at an angle $+\theta_{X Y}$ in the $X Y$ plane of the sample. This produces a GRM in the $Z$ axis if the sample contains anisotropically distributed SSD ferrimagnetic particles in the $X Y$ plane. 
but quite time consuming, taking a few hours to perform. It is effectively the remanence equivalent of the anisotropy of magnetic susceptibility (AMS) delineator (see the AMS section below), since it measures the differences in anisotropy of the SSD ferro- or ferrimagnetic particles in a particular plane, and can identify the orientations of the maximum and minimum SSD anisotropy axes within that plane. The GRM method does not, however, give the magnitudes of those axes.

\subsection{Anisotropy of Isothermal Remanent Magnetization (AIRM)}

This method applied a direct field (DF) to the sample to produce an isothermal remanent magnetization (IRM). This was achieved by placing the sample inside a pulse magnetizer, and applying a DF of about $100 \mathrm{~ms}$ duration. The resulting IRM components $(x, y$ and $z$ ) were then measured in a spinner magnetometer. This procedure was undertaken in 3 orthogonal directions along the $X, Y$, and $Z$ sample axes. This produced a total of 9 tensor components from which a $3 \mathrm{D}$ AIRM ellipsoid could be calculated giving the magnitudes and directions of the 3 principal anisotropy axes [3] [7]. The sample was tumble AF demagnetized before each magnetization step.

The pulse magnetization was carried out at two different DF strengths, $20 \mathrm{mT}$ and $60 \mathrm{mT}$, in order to determine the anisotropy of different ferrimagnetic particle size populations. The higher field affects the SSD ferrimagnetic particles as well as the MD ferrimagnetic particles, whereas the lower field only affects the larger MD ferrimagnetic particles (the lower field is below the anisotropy field of the SSD particles). A typical AIRM determination from the 3 magnetization and 3 demagnetization steps takes around 30 minutes.

\subsection{Anisotropy of Magnetic Susceptibility (AMS)}

This method determines a 3D AMS ellipsoid for the sample. The sample was placed in an anisotropy delineator and rotated successively in 3 orthogonal planes in a low $(500 \mu \mathrm{T})$ magnetic field. If the sample is anisotropic a sinusoidal voltage of twice the rotation frequency is induced in pick up coils. The amplitude of the voltage is proportional to the difference between the maximum and minimum susceptibilities in the sample plane perpendicular to the rotation axis. By adding a bulk magnetic susceptibility value to the anisotropy delineator results the magnitudes and orientations of the 3 principal AMS axes can be determined. The bulk value was measured along the $Z$ sample axis (see Figure 1 and Figure 2) using a magnetic susceptibility bridge. The sample is inserted into the coil of the bridge, which changes the coil's inductance, and this is proportional to the sample's magnetic susceptibility. The 3D AMS measurements are very rapid, with the anisotropy delineator measurements taking about a minute and the bulk susceptibility measurement taking a few seconds (with a background reading followed by a sample reading).

\subsection{Observed Petrofabric}

The visible rock anisotropy (petrofabric) was observed using a hand lens. This 
visible petrofabric reflected the alignment of the larger component grains (plagioclase and quartz) of the sample.

\subsection{Anisotropy of Shear Wave Velocity (ASWV)}

2D measurements of shear wave anisotropy were possible in the $X Y$ plane of the sample. Two parallel planar transducers were placed on the flat end faces (which were oriented in the $X Y$ plane) of the cylindrical sample plug. Whilst the overall wave train propagated along the axis of the plug (the $Z$ axis), the shear waves had a transverse motion in the perpendicular $X Y$ plane. By rotating the sample plug in the $X Y$ plane with respect to the transducers, as per the method described in [8], it was possible to detect shear wave anisotropy in the $X Y$ plane. A shear wave was propagated through the sample by one of the transducers and detected by the other, and the shear wave arrival time was recorded. Then the sample was rotated through $22.5^{\circ}$ in the $X Y$ plane and a shear wave was again propagated through the sample. This process was repeated every $22.5^{\circ}$ as the $X Y$ plane was rotated about $360^{\circ}$. The shear wave arrival times were recorded, and the velocities determined at each orientation to create a $2 \mathrm{D}$ plot of the shear wave velocity anisotropy in the XY plane.

\section{Results and Discussion}

\subsection{Gyroremanent Magnetization (GRM) Anisotropy}

Application of the AF at various angles in the $X Y, Y Z$ and $Z X$ planes of the sample caused GRM components to be acquired perpendicular to each plane along the $Z, X$ and $Y$ axes respectively (Figures 3-5) consistent with GRM theory [1]. Figures 3-5 demonstrate that the variation in magnitude of the GRM largely followed a $\sin (2 \theta)$ shape in each case (from the red fitting curves), where $\theta$ was the orientation of the applied AF as shown in Figure 2, in accordance with GRM theory [1]. This was in contrast to some previous experimental results for a sample comprising magnetic tape [1] which exhibited a "distorted" $\sin (2 \theta)$ curve. The SSD particles in the magnetic tape sample from [1] were highly interacting, since they were densely coated on the plastic tape. In contrast, the SSD particles in our natural rock sample are expected to be more dispersed and therefore less interacting. This may explain why we obtained undistorted $\sin (2 \theta)$ GRM curves for our natural sample, similar to results for man-made samples containing dispersed (non-interacting) SSD particles [5].

The GRM curves allow one to determine the predominant alignment of the SSD particles within each plane. Figure 3 shows that the z-component of the GRM was negative when the AF was oriented at $\theta=-90^{\circ}$ to $-15^{\circ}$ and when $\theta=$ $75^{\circ}$ to $90^{\circ}$ in the $X Y$ plane, and positive when the AF was oriented at $\theta=-15^{\circ}$ to $75^{\circ}$ in that plane (the equation of the best fit curve through the data points is $y(x)=50 \sin (2 x+\pi / 6)$ where $\mathrm{x}$ is the horizontal axis and $\mathrm{y}$ is the vertical axis). According to GRM theory [1] this indicates that the SSD ferrimagnetic particles in the rock sample are aligned closer to the $X$ axis (oriented at $\theta=0^{\circ}$ ) than the $Y$ 


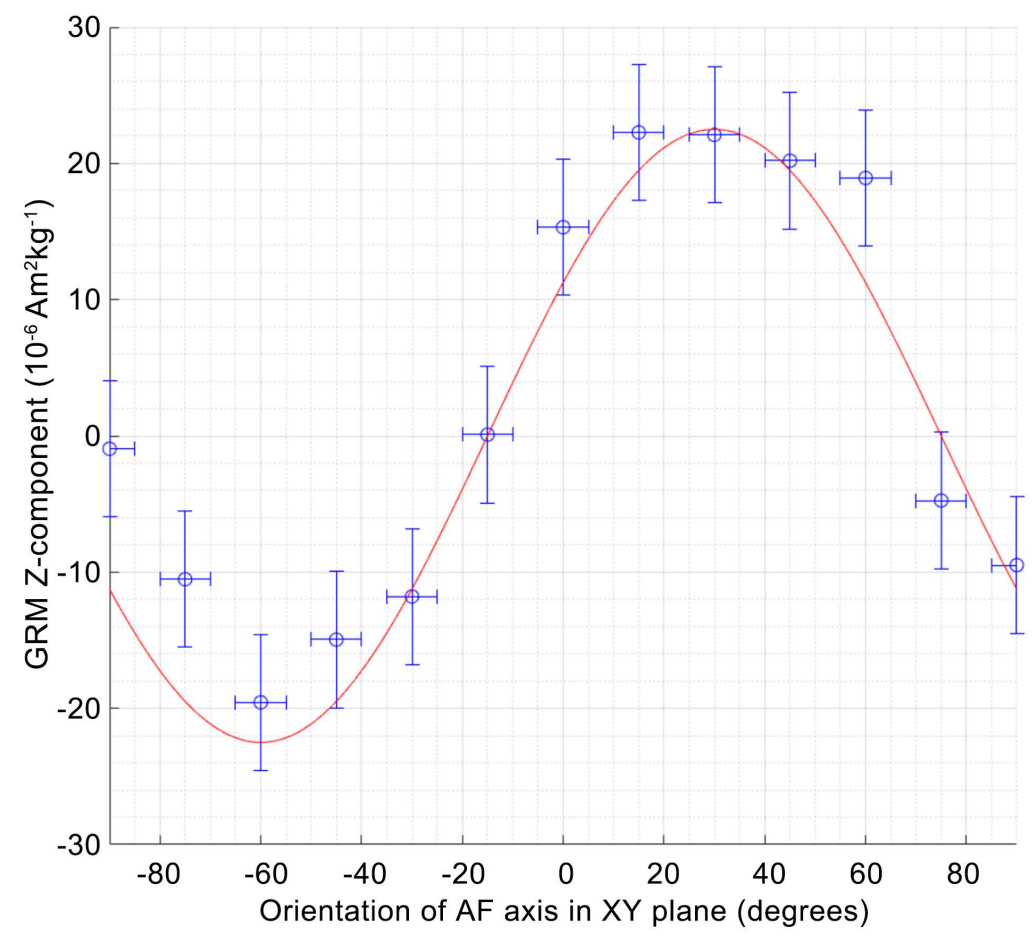

Figure 3. The z-components of GRM for orientations of the AF axis in the $X Y$ plane. The results indicate that the maximum alignment axis of the SSD particles in the $X Y$ plane occurs at an AF orientation of $\theta=-15^{\circ}$ (the AF orientation where GRM is zero in the positive slope part of the GRM curve), close to the $X$ axis.

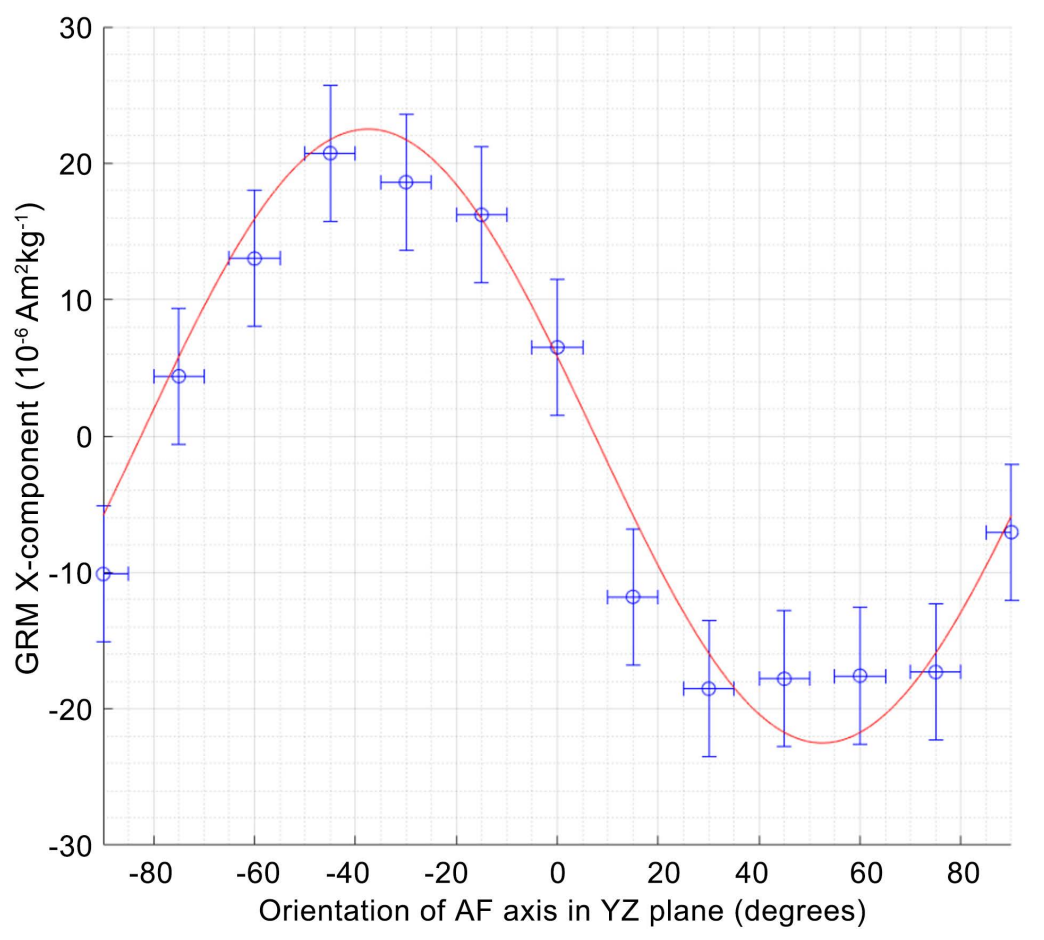

Figure 4. The $\mathrm{x}$-components of GRM for orientations of the AF axis in the $Y Z$ plane. The results indicate that the maximum alignment axis of the SSD particles in the $Y Z$ plane occurs at an AF orientation of $\theta=-82.5^{\circ}$ or $97.5^{\circ}$ (the AF orientation where GRM is zero in the positive slope part of the GRM curve), close to the $Z$ axis. 


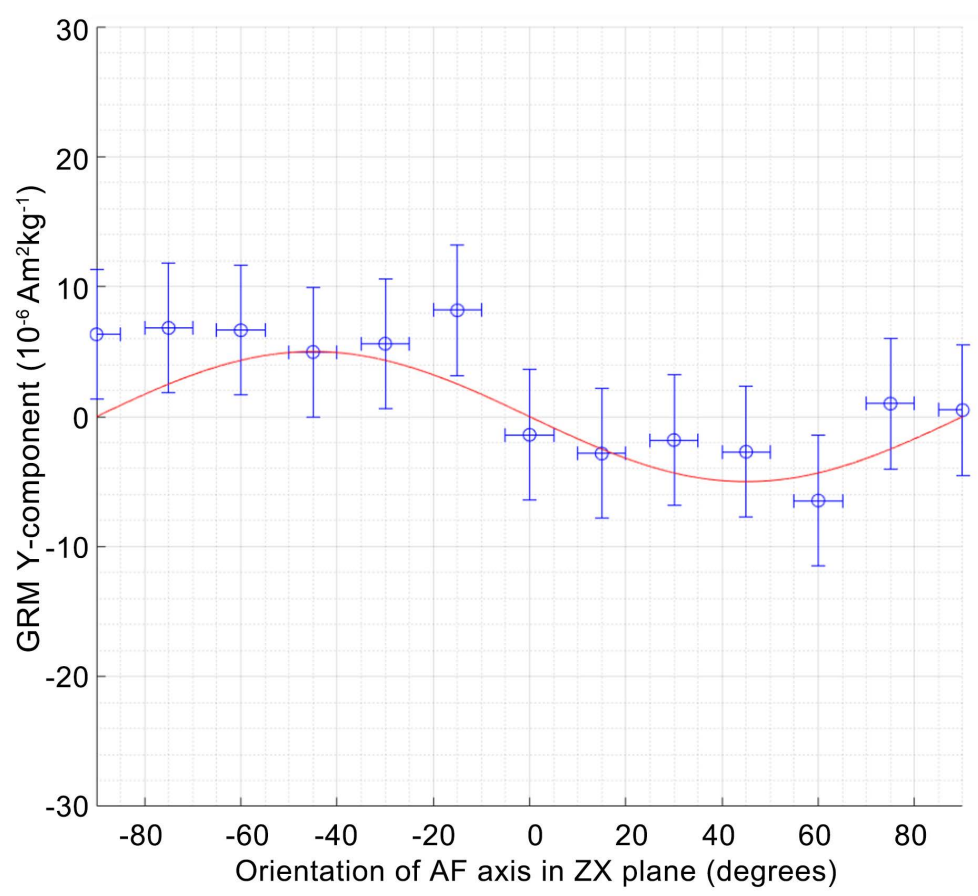

Figure 5. The $y$-components of GRM for orientations of the AF axis in the $Z X$ plane. The results indicate that the maximum alignment axis of the SSD particles in the $Z X$ plane occurs at an AF orientation of $\theta=-90^{\circ}$ or $90^{\circ}$ (the AF orientation where GRM is zero in the positive slope part of the GRM curve), identical to the $X$ axis.

axis (oriented at $\theta=-90^{\circ}$ or $90^{\circ}$ ). The maximum principal (i.e., preferred alignment) axis of the SSD particles in the $X Y$ plane occurs at an AF orientation of $\theta=-15^{\circ}$ (the AF orientation where the GRM is zero in the positive slope part of the GRM curve), which is close to the $\mathrm{X}$ axis.

Figure 4 shows that the $x$-component of the GRM was negative when the AF was oriented at $\theta=-90^{\circ}$ to $-82.5^{\circ}$ and when $\theta=7.5^{\circ}$ to $90^{\circ}$ in the $Y Z$ plane, and positive when the AF was oriented between $\theta=-82.5^{\circ}$ to $7.5^{\circ}$ in that plane (the equation of the best fit curve through the data points is $y(x)=50 \sin (2 x+\pi / 12))$. According to GRM theory [1] this indicates that the SSD ferrimagnetic particles in the rock sample are aligned closer to the $Z$ axis (oriented at $\theta=-90^{\circ}$ or $90^{\circ}$ in this case) than the $Y$ axis (oriented at $\theta=0^{\circ}$ ). The maximum principal axis of the SSD particles in the $Y Z$ plane occurs at an AF orientation of $\theta=-82.5^{\circ}$ or $97.5^{\circ}$ (the AF orientation where the GRM is zero in the positive slope part of the GRM curve), which is very close to the $\mathrm{Z}$ axis.

Likewise Figure 5 shows that the $y$-component of the GRM was negative when the $\mathrm{AF}$ was oriented at $\theta=0^{\circ}$ to $90^{\circ}$ in the $Z X$ plane, and positive when the AF was oriented at $\theta=-90^{\circ}$ to $0^{\circ}$ (the equation of the best fit curve through the data points is $y(x)=15 \sin (2 x)$ ). According to GRM theory [1] this indicates that the SSD ferrimagnetic particles are slightly more aligned along the $X$ axis (oriented at $\theta=-90^{\circ}$ or $90^{\circ}$ in the $Z X$ plane) than the $Z$ axis (oriented at $\theta=0^{\circ}$ ) given that the GRM magnitudes in Figure 5 are much less than those for the previous two planes shown in Figure 3 and Figure 4. The maximum principal axis of the SSD 
particles in the $Z X$ plane occurs at an orientation of $\theta=-90^{\circ}$ or $90^{\circ}$ (the AF orientation where the GRM is zero in the positive slope part of the GRM curve), which is identical to the $X$ axis in the $Z X$ plane.

Thus the combined GRM results indicate that the maximum principal anisotropy axis of the SSD ferrimagnetic particles (i.e., the preferential alignment axis) is close to the $X$ sample axis, the intermediate principal anisotropy axis is close to the $Z$ sample axis, and the minimum principal anisotropy axis is close to the $Y$ sample axis.

\subsection{Comparison with Other 3D Anisotropy Results (AIRM and AMS)}

Table 1 shows the orientations (declinations and inclinations) and magnitudes (both the absolute and normalized magnitudes) of the three principal AIRM axes for both the $20 \mathrm{mT}$ and $60 \mathrm{mT}$ DF strengths. The orientations of the principal anisotropy axes determined at the two field strengths are very similar. This demonstrates that the SSD ferrimagnetic particles (which are preferentially affected by the higher field) are aligned in a very similar orientation to the MD ferrimagnetic particles (which are preferentially affected by the lower DF). The percentage AIRM, $100[(\max -\min ) /(\max +i n t+\min )]$, for a $\mathrm{DF}$ of $20 \mathrm{mT}$ is slightly larger at $26 \%$ than for a DF of $60 \mathrm{mT}$ at $18 \%$. This may be due to the MD ferrimagnetic particles being more aligned than the smaller SSD ferrimagnetic particles, or the MD ferrimagnetic particles may be slightly more elongated, or it could merely be due to the non-linear acquisition of IRM with applied field strength [7].

The orientations of the principal AIRM axes compare well with the GRM results.

Table 1. The magnitudes and orientations $(\mathrm{Dec}=$ Declination, $\mathrm{Inc}=$ Inclination $)$ of the 3 principal anisotropy axes of the rock sample from 3D Anisotropy of Isothermal Remanent Magnetization (AIRM) and Anisotropy of Magnetic Susceptibility (AMS) measurements.

\begin{tabular}{|c|c|c|c|c|c|}
\hline \multirow{2}{*}{$\begin{array}{l}\text { Anisotropy } \\
\text { Technique }\end{array}$} & \multicolumn{5}{|c|}{ Principal Axes } \\
\hline & Axes & $\begin{array}{l}\text { IRM Magnitude } \\
\left(10^{-3} \mathrm{Am}^{2} \mathrm{~kg}^{-1}\right)\end{array}$ & $\begin{array}{l}\text { Normalised } \\
\text { Magnitudes }\end{array}$ & $\operatorname{Dec}\left({ }^{\circ}\right)$ & $\operatorname{Inc}\left({ }^{\circ}\right)$ \\
\hline \multirow[t]{3}{*}{$\operatorname{AIRM}(20 \mathrm{mT})$} & Max. & 35.45 & 0.46 & 349.7 & 0.5 \\
\hline & Int. & 26.53 & 0.34 & 254.0 & 84.9 \\
\hline & Min. & 15.78 & 0.20 & 259.7 & -5.1 \\
\hline \multirow[t]{4}{*}{$\operatorname{AIRM}(60 \mathrm{mT})$} & Max. & 70.03 & 0.42 & 344.3 & 7.0 \\
\hline & Int. & 55.22 & 0.34 & 210.0 & 80.0 \\
\hline & Min. & 39.50 & 0.24 & 255.2 & -7.1 \\
\hline & & $\begin{array}{l}\text { Mass Magnetic } \\
\text { eptibility }\left(10^{-8} \mathrm{~m}^{3}\right.\end{array}$ & & & \\
\hline \multirow[t]{3}{*}{ AMS } & Max. & 2478.9 & 0.39 & 353.4 & -2.7 \\
\hline & Int. & 2172.8 & 0.35 & 291.0 & 84.1 \\
\hline & Min. & 1638.2 & 0.26 & 263.1 & -5.2 \\
\hline
\end{tabular}


Both techniques show that the orientations of the principal anisotropy axes are close to the $X, Y$ and $Z$ sample axes, with the $X$ axis being the maximum axis (largest IRM magnitude), the $Z$ axis intermediate, and the $Y$ axis being the minimum axis.

Table 1 also shows the orientations and magnitudes (absolute and normalized) of the three principal AMS axes. The orientations of the principal AMS axes compare well with the GRM results and AIRM principal axes. The AMS magnitudes were also consistent with the GRM and AIRM results. The $X$ axis had the largest magnetic susceptibility, the $Z$ axis was intermediate, and the $Y$ axis had the minimum magnetic susceptibility.

The AMS results represent the sum effect of all the minerals (diamagnetic, paramagnetic, ferrimagnetic etc.) in the sample. In our sample the AMS signal is dominated by the ferrimagnetic fraction. The percent AMS is slightly lower (at $13 \%)$ than for the AIRM results. This is likely due to the sample containing both SSD and MD ferrimagnetic particles. Uniaxial SSD ferrimagnetic particles have a maximum magnetic susceptibility perpendicular to the long axes of the particles, whereas MD ferrimagnetic particles have a maximum magnetic susceptibility along the long axes of the particles [7] [9]. Thus for a mixture of SSD and MD particles with the same alignment orientation (as the AIRM results show in Table 1) the AMS results from each set of particles can partially cancel (in the present sample) or substantially cancel each other out (in some other cases) thus reducing the \% AMS.

The main visible petrofabric lineations on the rock are oriented close to the $X$ axis (declination $=0^{\circ}$ or $360^{\circ}$ ) in the $X Y$ plane as seen in Figure 1 . The GRM results of Figure 3 indicated that the SSD ferrimagnetic particles are preferentially aligned in a very similar orientation at $\theta=-15^{\circ}$ (declination $=345^{\circ}$ for comparison with values in Table 1 ) in the $X Y$ plane. This relatively small difference in orientation between the visible rock fabric and the alignment of the SSD ferrimagnetic particles may not be significant given the uncertainty bars shown on the GRM plots. Alternatively, the small difference may be real if the SSD particles are not aligned exactly with the overall rock fabric of the larger plagioclase and quartz grains. The lineations in $X Y$ also appear to extend in the $Z$ axis, suggesting a somewhat planar fabric in the $Z X$ plane, consistent with the $X$ and $Z$ axes being the maximum and intermediate axes from the GRM, AIRM and AMS results.

\subsection{Anisotropy of Shear Wave Velocity and Comparison with Other Anisotropy Results in the XY Plane}

Since the shear wave velocity anisotropy measurements could only be performed in $2 \mathrm{D}$ in the $X Y$ plane we will compare the shear wave results with the visible petrofabric and GRM results in the $X Y$ plane. We will also compare this $2 \mathrm{D}$ data with the $3 \mathrm{D}$ data from the AIRM and AMS results. The $2 \mathrm{D}$ shear wave velocity results in the $X Y$ plane are plotted in Figure 6 and fit with a least squares fitting method, which generated an $8^{\text {th }}$ degree polynomial to fit the dataset. The results 


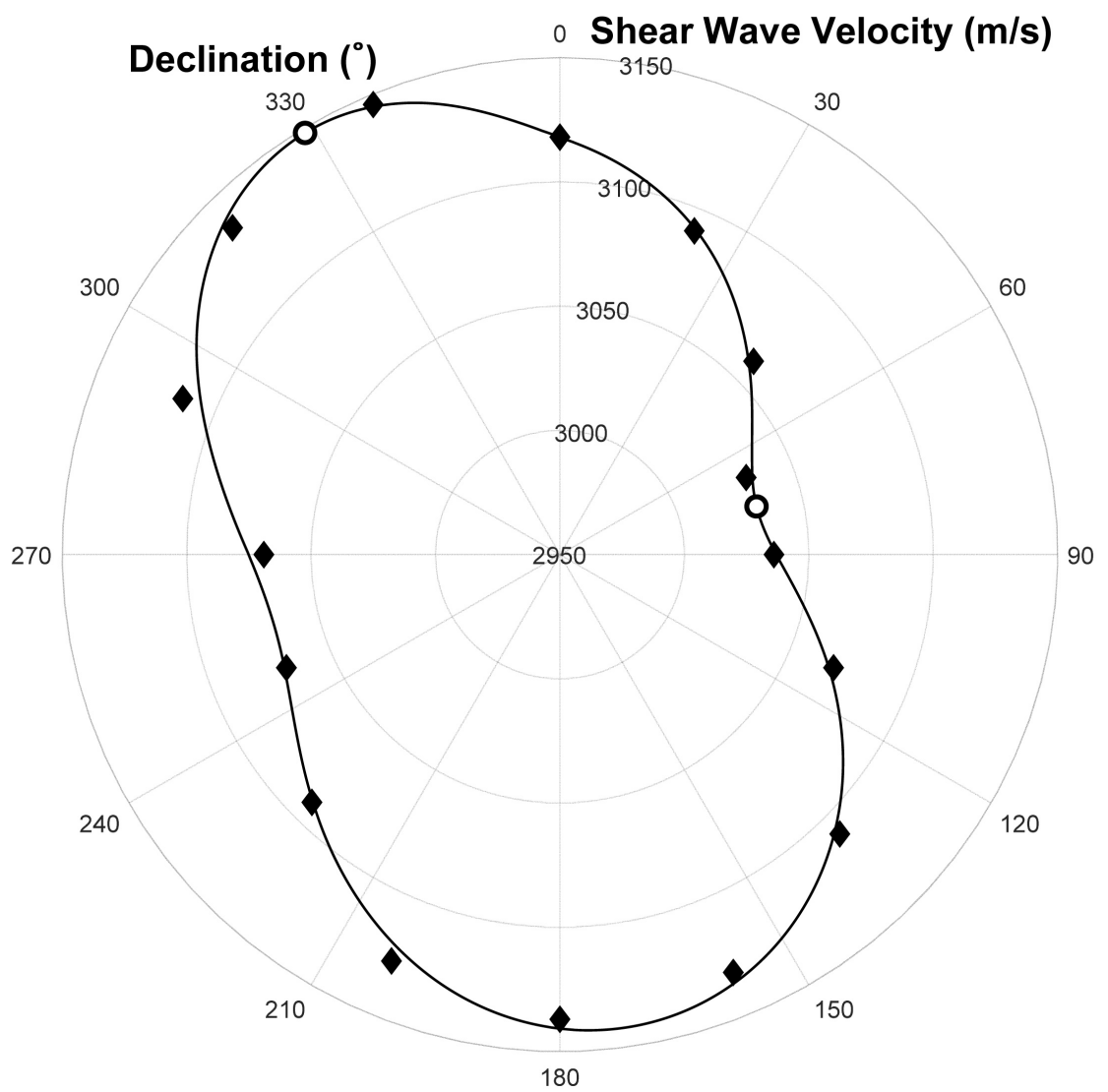

Figure 6. Shear wave velocity results at various orientations in the $X Y$ plane of the rock sample. Experimental data points (black diamonds) were fit with a least squares curve fitting program (black curve). The circles at declinations $329^{\circ}$ and $76^{\circ}$ are the maximum and minimum velocities respectively from the fitting curve.

form a "figure of 8 " pattern, demonstrating anisotropy in the $X Y$ plane. If the sample had been isotropic in the $X Y$ plane then the results would have formed a circle. From the best fit curve the declination of the maximum shear wave velocity axis was at $329^{\circ}$ (or $-31^{\circ}$ anticlockwise from $0^{\circ}$ ) with a magnitude of 3148 $\mathrm{m} / \mathrm{s}$, and the minimum axis was at $76^{\circ}$ with magnitude of $3030 \mathrm{~m} / \mathrm{s}$. It is not presently clear why the shear wave velocity results do not form a perfectly symmetrical "figure of 8 ". It might be due to slight differences in the coupling of the transducers to the sample during each experimental measurement.

The orientation of the maximum principal axis from the shear wave anisotropy results (declination $=-31^{\circ}$ or $329^{\circ}$ from the best fit curve) is not too far from that of the GRM results (declination $=-15^{\circ}$ or $345^{\circ}$ ). If one considers the maximum experimental shear wave value at a declination $=-22.5^{\circ}$ or $337.5^{\circ}$ (rather than the maximum value derived from the fitting curve) then the difference between the shear wave and GRM results is even less. The shear wave results show that the overall rock anisotropy from the main rock forming minerals (plagioclase and quartz) has quite a similar orientation to that of the small fraction of SSD ferrimagnetic particles (from the GRM results). One possible reason for the relatively small difference between the orientation of the maximum axis from 
the shear wave results compared to the GRM results could be the different sampling frequency in the $X Y$ plane (every $22.5^{\circ}$ for the acoustic measurements, and every $15^{\circ}$ for the GRM measurements). Alternatively, the difference could be real if the small fraction of SSD ferrimagnetic particles are not aligned in exactly the same orientation as the overall rock fabric. Whilst there is a close correspondence between the orientation results for the different methods for this sample (Table 2), it may not always be the case for other samples. The advantage of performing all the different methods described here is that it allows one to determine the anisotropy of the different mineral types and, in the case of the ferrimagnetic fraction, different particle size fractions (SSD versus MD etc.).

Table 2 shows a comparison of the results for all the anisotropy methods in the $X Y$ plane. The declination and inclination of the maximum and minimum principal axes is quite similar for all the methods. For the results based on $2 \mathrm{D}$ data the inclinations are nominally $0^{\circ}$ since no information in the third dimension is included. For the results based on $3 \mathrm{D}$ methods we give the maximum and minimum principal axes (which were very close to the $X Y$ plane) based on the full 3D data, and so these determinations include small inclinations. The results demonstrate that the orientations of the fine SSD and MD ferrimagnetic particles (from the GRM, AIRM and AMS results) are quite similar to those of the main rock forming minerals plagioclase and quartz (from the shear wave velocity anisotropy and visible petrofabric).

Table 2. The orientations of the maximum and minimum principal axes in the $X Y$ plane from the various anisotropy techniques. Negative declination values are anticlockwise from $0^{\circ}$ or $360^{\circ}$ (e.g., -10.3 is the same as 349.7 for comparison with Table 1 ), and are given for easy comparison with the GRM plots of Figures 3-5.

\begin{tabular}{cccc}
\hline $\begin{array}{c}\text { Anisotropy Technique in } X Y \\
\text { Plane }\end{array}$ & $\begin{array}{c}\text { Principal Axis in } X Y \\
\text { Plane }\end{array}$ & $\begin{array}{c}\text { Declination } \\
\text { (degrees) }\end{array}$ & $\begin{array}{c}\text { Inclination } \\
\text { (degrees) }\end{array}$ \\
\hline $\begin{array}{c}\text { Shear Wave Anisotropy from 2D } \\
\text { Measurements }\end{array}$ & Maximum & $-31^{\star}(-22.5)^{\dagger}$ & 0.0 \\
GRM at 60mT from 2D & Minimum & $76.0^{\star}(67.5)^{\dagger}$ & 0.0 \\
Measurements & Minimum & -15.0 & 0.0 \\
Visual Petrofabric from 2D & Maximum & 75.0 & 0.0 \\
Observations & Minimum & 0.0 & 0.0 \\
AIRM at 20 mT from 3D & Maximum & -10.3 & 0.0 \\
Measurements & Minimum & 79.7 & 0.5 \\
AIRM at 60 mT from 3D & Maximum & -15.7 & -5.1 \\
Measurements & Minimum & 75.2 & 7.0 \\
& Maximum & -6.6 & -7.1 \\
AMS from 3D Measurements & Minimum & 83.1 & -2.7 \\
& & -5.2 \\
\hline
\end{tabular}

*From the shear wave best fit curve. ${ }^{\dagger}$ From the individual shear wave velocity values.

\section{Conclusions}

1) The magnitudes of the primary GRM components for the natural rock 
sample studied here exhibited a $\sin (2 \theta)$ dependence on AF axis orientation in respective perpendicular planes. This was consistent with theory [1] and previous experiments on man-made samples [5] for non-interacting SSD particles, and contrary to the distorted $\sin (2 \theta)$ experimental GRM curve previously reported for interacting SSD particles on magnetic recording tape [1].

2) The sign of the GRM with AF orientation in the $X Y, Y Z$ and $Z X$ planes indicated that the magnitudes of the three principal axes of the SSD particles in the rock sample were in the order $X>Z>Y$.

3) The orientations of the principal anisotropy axes from the GRM results were consistent with those determined from anisotropy of isothermal remanent magnetization (AIRM) and indicated that the alignment of MD ferrimagnetic particles was similar to that of the smaller SSD ferrimagnetic particles. Anisotropy of magnetic susceptibility (AMS), which measures the combined effect of all the minerals in the sample, but is dominated in this case by the ferrimagnetic particles, also gave principal axes whose orientations were consistent with those from the GRM and AIRM results.

4) The orientation of the overall rock anisotropy of the main rock forming minerals (dominated by plagioclase and quartz), as determined by both the visual petrofabric and quantitative shear wave velocity anisotropy results, was also consistent with the magnetic anisotropy results (GRM anisotropy, AIRM and AMS, which were all dominated by the ferrimagnetic particles). This indicated that the main rock forming minerals for the sample studied were aligned in quite similar orientations to the smaller amount of fine grained ferrimagnetic particles.

5) The results from all methods indicated that the sample is significantly anisotropic, and is not "quasi-isotropic" as suggested from acoustic measurements by a previous study [4].

\section{Acknowledgements}

D. K. P. thanks the Natural Sciences and Engineering Research Council of Canada (NSERC) for a Discovery Grant.

\section{Conflicts of Interest}

The authors declare no conflicts of interest regarding the publication of this paper.

\section{References}

[1] Stephenson, A. (1980) Nature, 284, 49-51. https://doi.org/10.1038/284049a0

[2] Edwards, J. (1982) Geophysical Journal of the Royal Astronomical Society, 71, 199-214. https://doi.org/10.1111/j.1365-246X.1982.tb04993.x

[3] Potter, D.K. (2004) A Comparison of Anisotropy of Magnetic Remanence Methods-A User's Guide for Application to Paleomagnetism and Magnetic Fabric Studies. In: Martin-Hernandez, F., Luneburg, C.M., Aubourg, C. and Jackson, M., Eds., Magnetic Fabric. Methods and Applications, Geological Society, London, Spe- 
cial Publications, 21-35.

[4] Cholach, P.Y., Molyneux, J.B. and Schmitt, D.R. (2005) Canadian Journal of Earth Sciences, 42, 533-554. https://doi.org/10.1139/e04-094

[5] Stephenson, A. and Potter, D.K. (1987) IEEE Transactions on Magnetics, 23, 3820-3830. https://doi.org/10.1109/TMAG.1987.1065524

[6] Stephenson, A. (1981) Physics of the Earth and Planetary Interiors, 25, 163-166. https://doi.org/10.1016/0031-9201(81)90149-7

[7] Stephenson, A., Sadikun, S. and Potter, D.K. (1986) Geophysical Journal of the Royal Astronomical Society, 84, 185-200. https://doi.org/10.1111/j.1365-246X.1986.tb04351.x

[8] Snow, B.C. (2018) Nanoparticle Magnetic Characterization and Comparison of Rock Anisotropy Methods. MSc Thesis, University of Alberta, Alberta, 95.

[9] Potter, D.K. and Stephenson, A. (1988) Geophysical Research Letters, 15, 1097-1100. https://doi.org/10.1029/GL015i010p01097

\section{Nomenclature}

AF: Alternating Field

AIRM: Anisotropy of Isothermal Remanent Magnetization

AMS: $\quad$ Anisotropy of Magnetic Susceptibility

AMR: $\quad$ Anisotropy of Magnetic Remanence

ASWV: $\quad$ Anisotropy of Shear Wave Velocity

DF: $\quad$ Direct Field

GRM: $\quad$ Gyroremanent Magnetization

IRM: Isothermal Remanent Magnetization

MD: $\quad$ Multidomain

NRM: $\quad$ Natural Remanent Magnetization

SSD: $\quad$ Stable Single Domain

$X, Y, Z: \quad$ The $X, Y$ and $Z$ axes of the sample (see Figure 1 and Figure 2)

$X Y, Y Z, Z X$ : The $X Y, Y Z$ and $Z X$ planes of the sample

$x, y, Z$ The $x, y$ and $z$ components of magnetic remanence 